\title{
Guidelines for the Diagnosis and Management of Critical IIIness-Related Corticosteroid Insufficiency (CIRCI) in Critically III Patients (Part I): Society of Critical Care Medicine (SCCM) and European Society of Intensive Care Medicine (ESICM) 2017
}

\author{
Djillali Annane, MD, PhD ${ }^{1}$; Stephen M. Pastores, MD, FCCM²; Bram Rochwerg, $\mathrm{MD}^{3}$; \\ Wiebke Arlt, MD, DSc, FRCP ${ }^{4}$; Robert A. Balk, MD, MCCM5; Albertus Beishuizen, MD, $\mathrm{PhD}^{6}$; \\ Josef Briegel, $\mathrm{MD}, \mathrm{PhD}^{7}$; Joseph Carcillo, $\mathrm{MD}, \mathrm{FCCM}^{8}$; Mirjam Christ-Crain, $\mathrm{MD}, \mathrm{PhD}^{9}$; \\ Mark S. Cooper, MD $^{10}$; Paul E. Marik, MD, FCCM ${ }^{11}$; Gianfranco Umberto Meduri, MD ${ }^{12}$; \\ Keith M. Olsen, PharmD, FCCM ${ }^{13}$; Sophia C. Rodgers, RN, MSN, ACNP, FCCM ${ }^{14}$; James A. Russell, MD ${ }^{15}$; \\ Greet Van den Berghe, MD, $\mathrm{PhD}^{16}$
} ${ }^{1}$ General ICU Department, Raymond Poincaré Hospital (APHP), Health
Science.

${ }^{2}$ Department of Anesthesiology and Critical Care Medicine, Memorial Sloan Kettering Cancer Center, New York, NY, USA.

${ }^{3}$ Division of Critical Care, Department of Medicine, McMaster University, Hamilton, ON, Canada.

${ }^{4}$ Institute of Metabolism and Systems Research (IMSR), University of Birmingham and Centre for Endocrinology, Diabetes and Metabolism (CEDAM), Birmingham Health Partners, Birmingham, UK.

${ }^{5}$ Division of Pulmonary and Critical Care Medicine, Rush University Medical Center, Chicago, IL, USA.

${ }^{6}$ Department of Intensive Care Medicine, Medisch Spectrum Twente, Enschede, The Netherlands.

${ }^{7}$ Anesthesiology and Critical Care Medicine, Klinik für Anästhesiologie, Klinikum der Universität, Munich, Germany.

${ }^{8}$ Department of Critical Care Medicine and Pediatrics, University of Pittsburgh School of Medicine, Pittsburgh, PA, USA.

${ }^{9}$ Department of Endocrinology, Diabetology and Metabolism, Clinical Research, University Hospital Basel, Basel, Switzerland.

${ }^{10}$ Department of Endocrinology, Concord Hospital, University of Sidney, Sidney, NSW, Australia.

${ }^{11}$ Division of Pulmonary and Critical Care Medicine, Eastern Virginia Medical School, Norfolk, VA, USA.

${ }^{12}$ Division of Pulmonary, Critical Care, and Sleep Medicine, Department of Medicine, Memphis Veterans Affairs Medical Center, Memphis, TN, USA.

${ }^{13}$ College of Pharmacy, University of Arkansas for Medical Sciences, Little Rock, AR, USA.

${ }^{14}$ Clinical Adjunct Faculty, University of New Mexico and Sandoval Regional Medical Center, Albuquerque, NM, USA.

${ }^{15}$ Division of Critical Care Medicine, Centre for Heart Lung Innovation, St. Paul's Hospital, University of British Columbia, Vancouver, Canada.

Copyright (C) 2017 by the Society of Critical Care Medicine and European Society of Intensive Care Medicine. All Rights Reserved.

DOI: $10.1097 / C C M .0000000000002737$
${ }^{16}$ Clinical Division and Laboratory of Intensive Care Medicine, Department of Cellular and Molecular Medicine, KU Leuven University and Hospitals, Louvain, Belgium.

Dr. Djillali Annane and Dr. Stephen M. Pastores were co-chairs of the task force and contributed equally to this work.

Dr. Annane has been involved with research relating to this guideline. Dr. Pastores participates in the American College of Physicians: Speaker at ACP Critical Care Update Precourse, the American College of Chest Physicians (CHEST) (faculty speaker at Annual Congress), the American Thoracic Society (ATS): Moderator at Annual Meeting, the European Society of Intensive Care Medicine (EISCM) (co-chair of Corticosteroid Guideline in collaboration with SCCM), and the Korean Society of Critical Care Medicine (co-director and speaker at Multiprofessional Critical Care Board Review Course). He has spoken on the topic of corticosteroid use in critical illness and specifically in sepsis at the International Symposium in Critical and Emergency Medicine in March 2017. Dr. Arlt participates in the Society for Endocrinology UK (Chair of the Clinical Committee, member of Council, member of the Nominations Committee) and the Endocrine Society USA (member, Publication Core Committee). Dr. Briegel participates in the European Society of Intensive Care Medicine, the Deutsche interdisziplinäre Vereinigung Intensivmedizin, and the Deutsche Gesellschaft für Anästhesie und Intensivmedizin, and he has given lectures and talks on hydrocortisone treatment of septic shock. Dr. Cooper participates in a range of specialist societies relating to endocrinology and bone disease. Dr. Meduri disclosed he is a government employee. Dr. Olsen participates in the American College of Clinical Pharmacy (grant review committee), and he represents the American Society of Health-System Pharmacists on the National Quality Forum for Surgery Measures. Dr. Rochwerg disclosed he is a methodologist for ATS, CBS, ESCIM, ASH. The remaining authors have disclosed that they do not have any potential conflicts of interest.

Supplemental digital content is available for this article which includes evidence profiles.

This article is being simultaneously published in Critical Care Medicine and Intensive Care Medicine. ICM DOI: 10.1007/s00134-017-4919-5

This article is linked to another article entitled "Critical Illness-Related Corticosteroid Insufficiency (CIRCI): A Narrative Review From a Multispecialty 
Task Force of the Society of Critical Care Medicine (SCCM) and the European Society of Intensive Care Medicine (ESICM)" published in parallel. For more information about this article, Email: pastores@mskcc.org

Objective: To update the 2008 consensus statements for the diagnosis and management of critical illness-related corticosteroid insufficiency $(\mathrm{CIRCl})$ in adult and pediatric patients.

Participants: A multispecialty task force of 16 international experts in critical care medicine, endocrinology, and guideline methods, all of them members of the Society of Critical Care Medicine and/ or the European Society of Intensive Care Medicine.

Design/Methods: The recommendations were based on the summarized evidence from the 2008 document in addition to more recent findings from an updated systematic review of relevant studies from 2008 to 2017 and were formulated using the Grading of Recommendations Assessment, Development, and Evaluation (GRADE) methodology. The strength of each recommendation was classified as strong or conditional, and the quality of evidence was rated from high to very low based on factors including the individual study design, the risk of bias, the consistency of the results, and the directness and precision of the evidence. Recommendation approval required the agreement of at least $80 \%$ of the task force members.

Results: The task force was unable to reach agreement on a single test that can reliably diagnose $\mathrm{CIRCl}$, although delta cortisol (change in baseline cortisol at $60 \mathrm{~min}$ of $<9 \mu \mathrm{g} / \mathrm{dL}$ ) after cosyntropin $(250 \mu \mathrm{g})$ administration and a random plasma cortisol of $<10 \mu \mathrm{g} / \mathrm{dL}$ may be used by clinicians. We suggest against using plasma-free cortisol or salivary cortisol level over plasma total cortisol (conditional, very low quality of evidence). For treatment of specific conditions, we suggest using IV hydrocortisone $<400 \mathrm{mg} /$ day for $\geq 3$ days at full dose in patients with septic shock that is not responsive to fluid and moderate- to high-dose vasopressor therapy (conditional, low quality of evidence). We suggest not using corticosteroids in adult patients with sepsis without shock (conditional recommendation, moderate quality of evidence). We suggest the use of IV methylprednisolone $1 \mathrm{mg} / \mathrm{kg} /$ day in patients with early moderate to severe acute respiratory distress syndrome $\left(\mathrm{PaO}_{2} / \mathrm{FiO}_{2}<200\right.$ and within 14 days of onset) (conditional, moderate quality of evidence). Corticosteroids are not suggested for patients with major trauma (conditional, low quality of evidence).

Conclusions: Evidence-based recommendations for the use of corticosteroids in critically ill patients with sepsis and septic shock, acute respiratory distress syndrome, and major trauma have been developed by a multispecialty task force. (Crit Care Med 2017; 45:2078-2088)

Key Words: corticosteroids; glucocorticoids; critical illness; sepsis; septic shock; acute respiratory distress syndrome; major trauma

\section{INTRODUCTION}

Critical illness-related corticosteroid insufficiency (CIRCI) is a concept that was first introduced in 2008 by an international multidisciplinary task force convened by the Society of Critical Care Medicine (SCCM) to describe impairment of the hypothalamic pituitary axis (stress response) during critical illness (1). CIRCI is characterized by dysregulated systemic inflammation resulting from inadequate intracellular glucocorticoid-mediated anti-inflammatory activity for the severity of the patient's critical illness. The putative symptoms of CIRCI are listed in Table 1. CIRCI is associated with increased circulating levels of biological markers of inflammation and coagulation over time, morbidity, length of ICU stay, and mortality. Given the growing body of evidence that CIRCI occurs across a broad spectrum of critical illness, an understanding of the pathogenesis and treatment of CIRCI is important to all critical care providers.

Two emerging themes made it necessary to revisit the concept, diagnosis, and management of CIRCI (1): the recognition of the importance of evidence-based approaches to patient care to enhance quality, improve safety, and establish a clear and transparent framework for service development and healthcare provision (2); the widespread use of corticosteroids in critically ill patients, highlighting the need for a valid, reliable, and transparent process of evaluation to support key decisions.

Against this background, the SCCM and the European Society of Intensive Care Medicine (ESICM) have updated the 2008 guidelines for the diagnosis and treatment of CIRCI. In addition to rigorous application of GRADE (Grading of Recommendations Assessment, Development, and Evaluation) methodology, the

\section{TABLE 1. Putative Signs and Symptoms of Critical Illness-Related Corticosteroid Insufficiency (CIRCl)}

\begin{tabular}{ll} 
Clinical & \\
\hline General & Fever, asthenia \\
\hline Neurological & Confusion \\
& Delirium \\
& Coma \\
\hline Cardiovascular & Hypotension refractory to fluid resuscitation \\
& Decreased sensitivity to catecholamines \\
& High cardiac index \\
\hline Digestive & Nausea \\
& Vomiting \\
& Intolerance to enteral nutrition \\
\hline Respiratory & Persistent hypoxia \\
\hline Laboratory & Hypoglycemia \\
& Hyponatremia \\
& Hyperkalemia \\
& $\begin{array}{l}\text { Metabolic acidosis } \\
\text { Hypereosinophilia }\end{array}$ \\
\hline Hemaging & pituitary gland or adrenal gland \\
\hline
\end{tabular}


recommendations in this document focus on patient-important outcomes and utility to clinicians in everyday practice. It was not intended to define a standard of care, and should not be interpreted as such. As with any clinical practice guideline, it should not be interpreted as prescribing an exclusive course of management. The guideline covers CIRCI in critically ill children and adults. It does not cover chronic adrenal insufficiency and does not apply to neonates, because the guideline panel felt these areas represented separate fields of expertise. This guideline focuses on the three disorders that most clinicians associate with CIRCI: sepsis/septic shock, acute respiratory distress syndrome, and major trauma.

\section{Composition of the Guideline Development Group}

A multispecialty task force of international experts in critical care medicine, endocrinology, and guideline methods was convened from the membership of the SCCM and the ESICM. The first in-person meeting was held during the SCCM Critical Care Congress in San Francisco, CA in January 2014, followed by several teleconferences and electronic-based discussion at regular intervals and another three in-person meetings during the annual SCCM Critical Care Congress in January 2015, February 2016, and January 2017. Members who were unable to participate in the in-person meetings were given the opportunity to provide electronic input, and meeting updates were circulated.

\section{Conflict of Interest Policy}

We required all guideline task force members to fill out a detailed declaration of interest statement including all current and future financial conflicts of interest (COI) as well as past interests, restricted to the 2 years immediately before joining the guideline development process. No task force members reported any financial COI related to the development and writing of the guideline. All members were allowed to participate in all discussions and had equal weight in formulating the statements or in voting. All were allowed equal involvement in data extraction and writing the rationales. We also allowed members to exclude themselves from discussion and voting around specific questions if they felt significant academic COI. There was no input or funding from industry to produce this guideline. The COI forms are available from the SCCM and ESICM and are updated on a regular basis.

\section{Question Development}

The task force members developed a list of questions structured in the Population, Intervention, Comparison, and Outcome (PICO) format regarding the diagnosis and treatment of CIRCI in various clinical conditions (Supplemental Digital Content 1, http://links.lww.com/CCM/C914). The methods chair (BR) assisted in developing the PICO questions, i.e., framing the clinical questions in a searchable format. This required careful specification of the patient group $(\mathrm{P})$, the intervention $(\mathrm{I})$, the comparator $(\mathrm{C})$, and the outcomes $(\mathrm{O})$ for intervention questions and the patient group, index tests, reference standard, and target condition for questions of diagnostic test accuracy. For each question the task force agreed upon explicit review question criteria including study design features. Some of these questions had been previously addressed in the 2008 guidelines (1) and required updates of the evidence summaries, whereas others required de novo systematic reviews.

\section{Assessment of Relative Importance of Outcomes}

For each intervention question a list of outcomes was compiled, reflecting both benefits and harms of alternative management strategies. Outcomes (from the perspective of a patient) were ranked from "low" to "critical" importance and agreed by consensus of the task force members (Supplemental Digital Content 2, http://links.lww.com/CCM/C915). Ranking outcomes by their relative clinical importance helps to focus on those that are most relevant to patients and may lead to improved clarification during potential disagreements in decision making.

\section{SEARCHING FOR EVIDENCE}

\section{Sources}

The information technologists (based at McMaster University, Hamilton, Ontario) searched The Cochrane Database of Systematic Reviews, DARE, CENTRAL, and Medline for all PICO questions on diagnosis and treatment. All searches were updated through May 2017. The search strategies combined subject headings and text words for the patient population, index test and target condition for the diagnostic questions and subject headings and text words for the population and intervention for the intervention questions (Supplemental Digital Content 1, http://links.lww.com/CCM/C914).

If a previous meta-analysis of high quality was identified which addressed one of the PICO questions, this was used or updated to incorporate new evidence since its publication. Search and screening results were provided to the task force to ensure no important trials were missed or erroneously included.

Reference lists from the included publications were screened to identify additional papers. The methods chair also searched guideline databases and organizations including the National Guideline Clearinghouse, Guidelines International Network, Guidelines Finder, Centre for Reviews and Dissemination, National Institute for Health and Care Excellence, and professional critical care and endocrinology societies for guidelines in order to screen the reference lists.

\section{Selection of Studies for Inclusion}

The information technologists screened all titles and abstracts to discard the clearly irrelevant articles. Task force members completed a second screening. References were allocated to pairs of reviewers for evaluation of eligibility. All abstracts that did not meet the inclusion criteria were discarded. Any discrepancies at this stage were resolved by group consensus. All pairs of reviewers, with help from the methods support team, retrieved full texts of potentially relevant studies and examined them independently for eligibility. Any discrepancies were resolved by consensus.

\section{Data Extraction and Critical Appraisal of Individual Studies}

For each included study, we collected relevant information on design, conduct, risk of bias, and relevant results. For each 
question, the methodologist extracted all individual study data and produced (when pooling was judged to be appropriate) forest plots by outcome. All analysis was done using Review Manager (RevMan) software version 5.3 (Copenhagen: The Nordic Cochrane Centre, The Cochrane Collaboration, 2014).

The risk of bias of the included studies was evaluated using various validated checklists, as recommended by the Cochrane Collaboration (2). These were AMSTAR for systematic reviews (3), the Cochrane Risk of Bias tool for randomized controlled trials (4), and the Newcastle-Ottawa scale for cohort and casecontrol studies (5).

\section{Evidence Profiles}

Evidence summaries for each question were prepared by the methodologist following the GRADE approach (6), using the GRADEpro Guideline Development Tool online software (www.gradepro.org).

The evidence profiles include the summary-pooled or narrative-outcome data, an absolute measure of intervention effect when appropriate, the importance of the outcome, and the summary of quality of evidence for each outcome. Evidence profiles were constructed by the methodologist and reviewed and confirmed with the rest of the task force members.

\section{Rating the Quality of the Evidence for Each Outcome Across Studies}

In accordance with GRADE, the task force initially categorized the quality of the evidence (certainty) for each outcome as high if it originated from randomized controlled trials (RCTs) and low if it originated from observational data. We subsequently downgraded the quality of the evidence by one or two levels if results from individual studies were at serious or very serious risk of bias, there were serious inconsistencies in the results across studies, the evidence was indirect, the data were sparse or imprecise, or publication bias was thought to be likely. If evidence arose from observational data, but effect sizes were large, there was evidence of a dose-response gradient, or all plausible confounding would either reduce a demonstrated effect or suggest a spurious effect when results showed no effect, we upgraded the quality of the evidence. By repeating this procedure, we obtained an overall quality of the evidence for each outcome and each intervention.

\section{Formulating Statements and Grading Recommendations}

Actionable recommendations. After the evidence summary tables and evidence profiles had been prepared, revised, and approved by the task force, the recommendations were finalized. All recommendations were developed based on the GRADE evidence profiles for each recommendation. Each of the following factors was considered in recommendation development: the quality of the evidence, the balance of desirable and undesirable consequences of compared management options, the assumptions about the patient's values and preferences associated with the decision, the implications for resource use and health equity, the acceptability of intervention to stakeholders, and the feasibility of implementation. Recommendations and their strength required the agreement of at least $80 \%$ of the task force members. Committee members unable to join the face-to-face meetings or teleconferences were provided opportunity for input electronically. The entire committee agreed on the final wording of each recommendation and rationale with further qualifications for each recommendation (e.g., subgroup considerations, justification, implementation considerations).

Each recommendation was designated either "strong" or "conditional" according to the GRADE approach (7). As outlined by GRADE, we used the phrasing "we recommend" for strong recommendations and "we suggest" for conditional (synonymous with the older term 'weak') recommendations (Table 2). The implications of the strength of the recommendations for patients, clinicians, and policy makers are shown in Table 3.

Writing Rationale. We collated the actionable recommendations and the clinical advice for each of the clinical questions in separate chapters structured according to a specific format. Each question resulted in one or more specific boxed statements. Within each recommendation the strength was indicated as strong or conditional and the quality of the supporting evidence as high, moderate, low or very low (Table 2).

\section{TABLE 2. Factors Determining Strong vs. Conditional Recommendation}

What Should be Considered

High or moderate evidence (Is there high or moderate quality evidence?)

Certainty about the balance of benefits vs. harms and burdens (Is there certainty?)

Recommended Process

The higher the quality of evidence, the more likely a strong recommendation

The larger the difference between the desirable and undesirable consequences and the certainty around that difference, the more likely a strong recommendation. The smaller the net benefit and the lower the certainty for that benefit, the more likely a weak recommendation.

Certainty in or similar values (/s there certainty or similarity?)

Resource implications (Are resources worth expected benefits?)
The more certainty or similarity in values and preferences, the more likely a strong recommendation.

The lower the cost of an intervention compared to the alternative and other costs related to the decision-i.e., fewer resources consumed-the more likely a strong recommendation. 


\begin{tabular}{llc} 
& Strong Recommendation & Conditional Recommendation \\
\hline For patients & $\begin{array}{c}\text { Most individuals in this situation would want the } \\
\text { recommended course of action and only a small } \\
\text { proportion would not. }\end{array}$ & $\begin{array}{c}\text { The majority of individuals in this situation would } \\
\text { want the suggested course of action, but } \\
\text { many would not. }\end{array}$ \\
\hline For clinicians & $\begin{array}{c}\text { Most individuals should receive the recommended } \\
\text { course of action. Adherence to this recommenda- } \\
\text { tion according to the guideline could be used as a } \\
\text { quality criterion or performance indicator. Formal } \\
\text { decision aids are not likely to be needed to help } \\
\text { individuals make decisions consistent with their } \\
\text { values and preferences. }\end{array}$ & $\begin{array}{c}\text { Different choices are likely to be appropriate } \\
\text { for different patients, and therapy should be } \\
\text { tailored to the individual patient's circum- } \\
\text { stances. Those circumstances may include } \\
\text { the patient or family's values and preferences. }\end{array}$ \\
\hline $\begin{array}{l}\text { The recommendation can be adapted as policy in most } \\
\text { situations including for the use as performance } \\
\text { indicators. }\end{array}$ & $\begin{array}{l}\text { Policy making will require substantial debates } \\
\text { and involvement of many stakeholders. } \\
\text { Policies are also more likely to vary between } \\
\text { regions. Performance indicators would } \\
\text { have to focus on the fact that adequate } \\
\text { deliberation about the management options } \\
\text { has taken place. }\end{array}$ \\
\hline
\end{tabular}

All statements are followed by advice for clinical practice, where relevant, and the rationale. The rationale contains a brief section on the relevant background and justification of the topic, followed by a short narrative review of the evidence.

\section{External Review}

External peer review was provided through the Board of Regents of the American College of Critical Care Medicine, the councils of the SCCM and ESICM, and the editorial boards of Critical Care Medicine and Intensive Care Medicine. Two international experts in endocrinology (George P. Chrousos, MD and Stefan R. Bornstein, MD) also reviewed the final draft of the guideline and provided comments.

\section{RECOMMENDATIONS FOR DIAGNOSIS OF CIRCI}

1. Is total cortisol response to synthetic adrenocorticotropic hormone (ACTH; cosyntropin) superior to random plasma or serum total cortisol for the diagnosis of CIRCI?

Recommendation: The task force makes no recommendation regarding whether to use delta cortisol (change in baseline cortisol at $60 \mathrm{~min}$ of $<9 \mu \mathrm{g} / \mathrm{dL})$ after cosyntropin $(250 \mu \mathrm{g})$ administration or a random plasma cortisol of $<10 \mu \mathrm{g} / \mathrm{dL}$ for the diagnosis of CIRCI.

Rationale: The 2008 guidelines suggested that the diagnosis of CIRCI is best made by a delta total serum cortisol of $<9 \mu \mathrm{g} /$ $\mathrm{dL}$ after IV cosyntropin $(250 \mu \mathrm{g})$ administration or a random total cortisol of $<10 \mu \mathrm{g} / \mathrm{dL}$ (1). To date, however, clinicians have not adopted these diagnostic criteria in their routine practice. Moreover, the latest Surviving Sepsis Campaign guidelines suggest not using the ACTH stimulation test to select patients with septic shock that may be treated with hydrocortisone (8).
Nevertheless, a recent guideline from the Endocrine Society confirmed that the high-dose $(250-\mu \mathrm{g})$ ACTH stimulation test is superior to other existing diagnostic tests to establish the diagnosis of primary adrenal insufficiency, with peak cortisol levels below $18 \mu \mathrm{g} / \mathrm{dL}$ (assay dependent) at 30 or $60 \mathrm{~min}$ indicating adrenal insufficiency (9).

We found one single-center randomized trial that compared low-dose ACTH $(1 \mu \mathrm{g})$ stimulation test with total random cortisol for diagnosis of adrenal insufficiency in 59 adults with septic shock (10). Compared with total random cortisol, the low-dose ACTH test was better able to predict a longer duration of vasopressor requirement and hemodynamic response to corticosteroids. Similarly, prospective cohort studies in adults with or without sepsis (11) and in patients with multiple trauma (12) found that patients with CIRCI, i.e., total cortisol levels $<10 \mu \mathrm{g} / \mathrm{dL}$ or delta cortisol $<9 \mu \mathrm{g} / \mathrm{dL}$, had poorer outcomes than patients without CIRCI. Likewise, a large multicenter prospective cohort study found that critically ill children with a delta cortisol $<$ $9 \mu \mathrm{g} / \mathrm{dL}$ after the low-dose ACTH stimulation test required higher-dose and prolonged treatment with catecholamines, a higher amount of fluid, and had a higher mortality rate (13). See Supplemental Digital Content 3 (http://links.lww. com/CCM/C916) for evidence profile.

Owing to the potential for risk of bias in study design, with only one single-center unblinded randomized trial and a small number of prospective cohort studies, and due to imprecision related to low numbers of patients included, we downgraded the quality of evidence to low. After two rounds of voting the task force members could not reach a consensus ( $>80 \%$ agreement) on whether the ACTH stimulation test is superior to random cortisol for the routine diagnosis of CIRCI. Due to the broad spectrum of abnormalities that may cause CIRCI, the task force thought it is unlikely that a single test can reliably diagnose CIRCI independent of its mechanisms, i.e., altered cortisol synthesis or metabolism, or tissue resistance to cortisol. 


\section{Is plasma or serum free cortisol level superior to plasma total cortisol level for the diagnosis of $\mathrm{CIRCl}$ ?}

Recommendation: We suggest against using plasma free cortisol level rather than plasma total cortisol for the diagnosis of CIRCI (conditional recommendation, very low quality of evidence).

Rationale: Free cortisol is the bioactive form of cortisol. Critically ill patients often present with low serum concentrations of cortisol-binding globulin (CBG) and hypoalbuminemia. In patients with low serum concentrations of cortisol binding proteins, serum total cortisol levels may not predict serum free cortisol levels, with a correlation between serum levels of free and total cortisol of only $50 \%$ to $60 \%$ (14).

We found no randomized trial that compared serum total versus free cortisol levels to diagnose CIRCI. A prospective study of 112 critically ill adults with treatment-insensitive hypotension, published after the 2008 recommendations, found a good correlation between serum concentrations of free and total cortisol before and after $250 \mu \mathrm{g}$ ACTH stimulation testing (15). These findings suggested that using total cortisol levels after ACTH testing is sufficient in critically ill adults. Another prospective cohort study of 69 critically ill patients to assess the time course of serum cortisol levels found that levels of both free and total cortisol predicted clinical outcomes (16). Another prospective cohort study of 29 adults with septic shock found remarkable differences between the serum concentrations of free and total cortisol levels both over time and in response to $1 \mu \mathrm{g}$ ACTH (17). See Supplemental Digital Content 3 (http:// links.lww.com/CCM/C916) for evidence profile.

Measurement of serum free cortisol levels involves cumbersome techniques that are unlikely to be available in all hospital laboratories and unlikely to provide a rapid turnaround time. There were a small number of low-quality observational studies with inconsistent findings. Thus, the task force suggested against measuring plasma free cortisol level over plasma total cortisol level in patients with suspected CIRCI.

\section{Is salivary free cortisol level superior to plasma total cortisol level for the diagnosis of $\mathrm{CIRCI}$ ?}

Recommendation: We suggest against using salivary rather than serum cortisol for diagnosing CIRCI (conditional recommendation, very low quality of evidence).

Rationale: In saliva, cortisol is found unbound. Thus, measuring salivary cortisol levels may inform on free cortisol levels and adrenal function. However, salivary cortisol levels may be impacted by a number of confounding factors such as gender, age, time and site of sampling, and saliva volume (18). A few studies evaluated the use of salivary cortisol as a measure for adrenal insufficiency. In one study, free cortisol level was more strongly correlated with salivary than with serum total cortisol in 88 cirrhotic patients (Spearman coefficient 0.91 and 0.76 , respectively; $p<0.001$ ) (19). In contrast, in a study of 57 patients with septic shock, there was no significant difference between free serum cortisol and salivary cortisol levels $(p=0.28)(20)$. In addition, the correlation between salivary cortisol and total serum cortisol levels was very good (80\%). Unbound plasma cortisol can be calculated using total serum cortisol and CBG measurements $(21,22)$. See Supplemental Digital Content 3 (http://links.lww.com/CCM/C916) for evidence profile.

The evidence demonstrating any benefit of using salivary cortisol over serum cortisol is extremely limited. Although salivary cortisol may be more closely correlated with free cortisol than total cortisol, no study has demonstrated that using salivary cortisol to diagnose CIRCI in critically ill patients leads to improved patient outcomes. Furthermore, the practicality and feasibility of using salivary cortisol is questionable given that it is tested by enzyme immunoassay, which may not be routinely available at most centers. Additionally, there are implementation concerns: in the Estrada-Y-Martin study (20), for example, 19 of the 57 patients were excluded because three initial samples did not provide any saliva, and 16 were eliminated owing to insufficient saliva or blood contamination. The task force therefore felt that using salivary cortisol would not be cost effective, practical, or feasible.

\section{Is the 1- $\mu \mathrm{g}$ ACTH stimulation test superior to the 250- $\mu$ g ACTH test for the diagnosis of $\mathrm{CIRCl}$ ?}

Recommendation: We suggest that the high-dose $(250-\mu \mathrm{g})$ rather than the low-dose $(1-\mu \mathrm{g})$ ACTH stimulation test be used for the diagnosis of CIRCI (conditional recommendation, low quality of evidence).

Rationale: The high-dose $(250-\mu \mathrm{g})$ ACTH stimulation test remains the most popular diagnostic test for adrenal insufficiency. However, this supraphysiologic dose of ACTH may result in significant stimulation of the adrenocortical cells in patients with proven adrenal insufficiency. Therefore, to increase the sensitivity of this diagnostic test, low-dose $(1-\mu \mathrm{g})$ ACTH was suggested. The high-dose ACTH test is easy to perform and safe. The low-dose ACTH test requires some preparation at the bedside as the commercial ampoules contain $250 \mu \mathrm{g}$ of ACTH.

A recent meta-analysis of 30 studies, involving 1209 adults and 228 children, found that for secondary adrenal insufficiency, the high- and low-dose ACTH tests had similar diagnostic accuracy (23). The likelihood ratio (LR) of a positive test was 9.1 and 5.9 for the high- and low-dose ACTH test, respectively, for adults and 43.5 and 7.7, respectively, for children. However, both tests had low sensitivity as suggested by the suboptimal LR of a negative test (adults: 0.39 and 0.19 for the high- and low-dose ACTH test, respectively; children: 0.65 and 0.34 , respectively). A prospective cohort study of 74 adults with septic shock found that the delta cortisol using the low- and high-dose ACTH tests was equally accurate in predicting vasopressor dependency and mortality (24). Likewise, in a prospective multicenter cohort study of critically ill children, the low- and high-dose ACTH tests showed similar accuracy in the prediction of clinical outcomes (13). See Supplemental Digital Content 3 (http://links.lww.com/CCM/ C916) for evidence profile.

Owing to easier practical modalities and the comparable accuracy of the low- and high-dose ACTH tests, the task force suggested using the high-dose rather than the low-dose ACTH test for the diagnosis of CIRCI. 


\section{Is hemodynamic response to hydrocortisone (50-300 mg) superior to the 250- $\mu$ g ACTH stimulation test for the diagnosis of $\mathrm{CIRCI}$ ?}

Recommendation: We suggest the use of the 250- $\mu$ g ACTH stimulation test rather than the hemodynamic response to hydrocortisone (50-300 mg) for the diagnosis of CIRCI (conditional recommendation, very low quality of evidence).

Rationale: Early reports on low-dose corticosteroids in human septic shock hypothesized that hemodynamic improvement unmasks adrenocortical insufficiency $(25,26)$. Hydrocortisone was found to improve the vasopressor response to norepinephrine in septic patients, this effect being more marked in patients with CIRCI (27). Arterial hypotension may serve as a useful marker of inadequate corticosteroid activity, although not all patients with septic shock may have CIRCI (28).

No studies are presently available that directly address this specific question. CIRCI diagnosed with the 250- $\mu$ g ACTH stimulation was associated with faster shock resolution in two studies $(29,30)$. In contrast, the CORTICUS trial found a similar hemodynamic response to corticosteroids in patients with or without CIRCI (31). The recent Hydrocortisone for Prevention of Septic Shock (HYPRESS) trial also did not find a difference in the development of septic shock in the presence or absence of CIRCI (32). However, in the HYPRESS trial only a limited number of patients were screened for CIRCI, affecting the reliability of these data. See Supplemental Digital Content 3 (http://links.lww.com/CCM/C916) for evidence profile.

Earlier shock resolution has been shown to lead to lower mortality (33). However, no studies compared the prognostic value of hemodynamic response to hydrocortisone versus the 250- $\mu \mathrm{g}$ ACTH test for the diagnosis of CIRCI. Meta-analyses examined only differences in mortality rates with corticosteroid treatment between those with and without documented CIRCI (34). Thus, the task force could only recommend the use of the $250-\mu \mathrm{g}$ ACTH stimulation test to diagnose CIRCI.

\section{Is corticotropin level superior to the $250-\mu \mathrm{g}$ ACTH stimulation test for the diagnosis of $\mathrm{CIRCI}$ ?}

Recommendation: We suggest against using corticotropin levels for the routine diagnosis of CIRCI (conditional recommendation, low quality of evidence).

Rationale: The plasma corticotropin level is determined by corticotropin release from the anterior pituitary gland into the systemic circulation. Normally, plasma concentrations of corticotropin and cortisol change in opposite directions. In primary adrenal insufficiency, plasma cortisol level is low and plasma corticotropin level is high. In hypopituitarism, plasma corticol level is low and plasma corticotropin level is low or normal. During critical illness, plasma corticotropin levels have been variably found to be low, normal, or high and likely follow a dynamic pattern with transiently elevated levels and subsequent decline over a period of weeks after the initial insult (1). We did not find any study that compared the diagnostic accuracy of corticotropin level with that of the ACTH stimulation test.

Owing to the complexity of measuring the plasma level of corticotropin, the task force deemed that it is not feasible in most institutions to obtain a corticotropin level with a sufficiently short turnaround time to have an impact on the acute management of the critically ill.

\section{RECOMMENDATIONS FOR CORTICOSTEROID USE IN CRITAL CARE CONDITIONS}

\section{Sepsis}

A. Should corticosteroids be administered among hospitalized adult patients with sepsis without shock?

Recommendation: We suggest against corticosteroid administration in adult patients with sepsis without shock (conditional recommendation, moderate quality of evidence).

Rationale: Sepsis and septic shock are major healthcare problems: they affect millions of people worldwide annually and are associated with mortality rates of $25-30 \%$ and high direct and indirect costs (35-39). Pro-inflammatory cytokines have been demonstrated to either suppress cortisol response to ACTH or compete with intracellular glucocorticoid function, which can result in CIRCI in septic patients. Sepsis-related CIRCI may in turn precipitate organ failure and result in lack of response to vasopressor therapy in these patients $(40,41)$. Thus, the potential benefit of corticosteroids for the treatment of sepsis has been tested in dozens of observational studies and trials over a period of several decades.

Analysis of 27 RCTs $(n=3176)$ of patients with sepsis with and without shock revealed a 28 -day mortality rate of $29.3 \%$ in patients receiving corticosteroids compared with $31.8 \%$ in those who received placebo (relative risk [RR] 0.87, 95\% CI $0.76-1.0)$ (42). The quality of evidence was considered low owing to inconsistency in the results and imprecision. See Supplemental Digital Content 4 (http://links.lww.com/CCM/ C917) for evidence profile.

A separate analysis of six RCTs $(n=826)$ of patients with sepsis without shock revealed a 28 -day mortality rate of $33.8 \%$ in patients receiving corticosteroids compared with $30.6 \%$ in those who received placebo (RR 1.11, 95\% CI 0.91-1.34) (42). Hyperglycemia was the most common adverse event, and corticosteroids did not increase the risk of secondary infections (RR $1.02,95 \%$ CI $0.87-1.20$ ). The quality of evidence was considered moderate due to imprecision, given the wide confidence intervals. See Supplemental Digital Content 4 (http://links.lww. com/CCM/C917) for evidence profile.

Most recently, the HYPRESS multicenter trial assigned patients with sepsis (excluding those with shock) to receive either a continuous infusion of $200 \mathrm{mg}$ of hydrocortisone for 5 days, followed by dose tapering until day $11(n=190)$, or placebo $(n=190)$ (33). The primary outcome was development of septic shock within 14 days. Patients who received hydrocortisone showed no difference in rates of progression to septic shock within 14 days from those given placebo (difference $-1.8 \%$; $95 \%$ CI $-10.7 \%$ to $7.2 \%$; $p=0.70$ ). In addition, there were no significant differences between the hydrocortisone and placebo groups for the use of mechanical ventilation $(53.2 \%$ vs $59.9 \%$ ), mortality at 28 days ( $8.8 \%$ vs $8.2 \%$ ) or up to 180 days (26.8\% vs $22.2 \%)$, ICU length of stay (median [interquartile 
range] 8 [5-15] vs 9 [6-17] days), or hospital length of stay (median [interquartile range] 26 [16-46] vs 25 [16-40] days). In the hydrocortisone versus placebo groups, $21.5 \%$ vs $16.9 \%$ had secondary infections, $8.6 \%$ vs $8.5 \%$ had ventilation weaning failure, $30.7 \%$ vs $23.8 \%$ had muscle weakness, and $90.9 \%$ vs $81.5 \%$ had hyperglycemia. Based on these results, the task force members agreed that corticosteroids may not be beneficial in adult patients with sepsis without shock.

\section{B. Should corticosteroids be administered among hospitalized adult patients with septic shock?}

Recommendation: We suggest using corticosteroids in patients with septic shock that is not responsive to fluid and moderate- to high-dose vasopressor therapy (conditional recommendation, low quality of evidence).

\section{What is the recommended dose and duration of treatment among hospitalized adult patients with septic shock treated with corticosteroids?}

Recommendation: If using corticosteroids for septic shock, we suggest using long course and low dose (e.g., IV hydrocortisone $<400 \mathrm{mg} /$ day for at $\geq 3$ days at full dose) rather than high dose and short course in adult patients with septic shock (conditional recommendation, low quality of evidence).

Rationale: The latest Cochrane systematic review of the use of low-dose hydrocortisone for treating septic shock, including 33 RCTs with a total of 4,268 patients (42), showed that corticosteroids significantly reduced the risk of death at 28 days compared with placebo. Three of these RCTs included children and the other 30 trials included only adults. Survival benefits were dependent on the dose of corticosteroids, with lower doses ( $<400 \mathrm{mg}$ of hydrocortisone or equivalent per day) for a longer duration of treatment ( 3 or more days at the full dose) found to be better, and on the severity of the sepsis. Furthermore, corticosteroids did not cause harm except for an increased incidence of hyperglycemia and hypernatremia; there was no increased risk of superinfection or gastrointestinal bleeding. See Supplemental Digital Content 4 (http://links.lww.com/ $\mathrm{CCM} / \mathrm{C} 917)$ for evidence profile.

A network meta-analysis of 22 trials suggested no clear evidence for the superiority of one type of corticosteroids over another in adult patients with septic shock (43). However, hydrocortisone boluses and infusions were more likely than methylprednisolone boluses and placebo to reverse shock.

Given the consistent effect of corticosteroids on shock reversal and the low risk for superinfection with low-dose corticosteroids, the task force suggests the use of low-dose IV hydrocortisone $<400 \mathrm{mg} /$ day for at least 3 days at full dose, or longer in adult patients with septic shock that is not responsive to fluid and moderate to high-dose ( $>0.1 \mu \mathrm{g} / \mathrm{kg} / \mathrm{min}$ of norepinephrine or equivalent) vasopressor therapy. The task force panel was unable to comment on pediatric patients with septic shock as the meta-analyses we reviewed did not include enough patients in this age group. A small pilot RCT (Steroids in Fluid and/or Vasoactive Infusion Dependent Pediatric Shock, STRIPES) demonstrated the feasibility of a larger RCT to address the role of corticosteroids for the treatment of pediatric shock (44). Since the publication of the Cochrane meta-analysis in 2015, a few small studies of early corticosteroid therapy in patients with pediatric septic shock and adult patients with sepsis-associated ARDS have been published (45-47) but the results are consistent with our current recommendations.

The Activated Protein C and Corticosteroids for Human Septic Shock (APROCCHSS) trial enrolled 1,241 adult patients with refractory septic shock from 35 centers in France (48). This trial commenced in 2008 and initially included the recombinant form of human activated protein C (APC), drotrecogin alfa-activated. The study featured a $2 \times 2$ factorial design with patients assigned to placebo of hydrocortisone + placebo of fludrocortisones + placebo of APC; hydrocortisone + fludrocortisone + placebo of APC; placebo of hydrocortisone + placebo of fludrocortisone + APC; or hydrocortisone + fludrocortisone + APC. Hydrocortisone was administered as a 50-mg IV bolus every $6 \mathrm{~h}$ and fludrocortisone as a $50-\mu \mathrm{g}$ tablet via a nasogastric tube once daily. In 2011, APC was withdrawn from the market after failing to demonstrate adequate efficacy in other clinical trials (49). Once APC was no longer available, the study continued without the APC arms; one arm then comprised placebo corticosteroids $(n=627)$ and the other arm comprised hydrocortisone and fludrocortisone combined $(n=614)$. Another large RCT (the ADRENAL study) conducted in Australia and New Zealand enrolled 3,800 patients either to hydrocortisone or to a placebo. Although enrolment is completed, results are not yet available (50). In this trial, no ACTH stimulation testing was performed. The final results of these two trials are still pending but once available may further define the role of corticosteroids in the setting of sepsis or septic shock. Our recommendations may have to be re-addressed once these results are available.

\section{Acute Respiratory Distress Syndrome}

Should corticosteroids be administered among hospitalized adult patients with acute respiratory distress syndrome?

Recommendation: We suggest use of corticosteroids in patients with early moderate to severe acute respiratory distress syndrome $\left(\mathrm{PaO}_{2} / \mathrm{FiO}_{2}\right.$ of $<200$ and within 14 days of onset $)$ (conditional recommendation, moderate quality of evidence).

Rationale: Acute respiratory distress syndrome (ARDS) represents an important public health problem globally. Despite advances in supportive care, ARDS is associated with a high mortality rate $(35 \%-45 \%)(51)$. ARDS is also associated with high costs of inpatient care and significant long-term morbidity and resource utilization (52). In ARDS, prolonged mechanical ventilation is associated with increased risk of disability and mortality at 1 year $(53,54)$.

Nine trials have investigated prolonged glucocorticoid treatment in ARDS (46). One of these trials was in patients with ARDS due to community-acquired pneumonia (59) and another was a subgroup analysis of the initial corticosteroid trial in septic shock (60). These trials consistently found that glucocorticoid treatment was associated with a significant reduction in markers of systemic inflammation (inflammatory cytokines and/or C-reactive protein levels), reduction in the duration of mechanical ventilation by approximately 7 days, and probable 
reduction in hospital mortality by approximately $7 \%$ and $11 \%$ in patients with mild and severe ARDS, respectively (moderate certainty) (61). All but two trials $(55,56)$ investigated treatment initiated in early ARDS. Compared with late ( $\geq 7$ days) initiation, early $(<72 \mathrm{~h})$ initiation of methylprednisolone treatment-when fibroproliferation (62) is still in the early stage of development (cellular with predominant type III procollagen)shows response to a lower daily methylprednisolone dose ( $1 \mathrm{mg} /$ $\mathrm{kg} /$ day vs $2 \mathrm{mg} / \mathrm{kg} /$ day) and is associated with faster disease resolution (e.g., shorter time to unassisted breathing, shorter time to ICU discharge) (61). See Supplemental Digital Content 4 (http://links.lww.com/CCM/C917) for evidence profile.

A recent individual patient data (IPD) analysis of the four largest trials $(n=322)$ investigating prolonged methylprednisolone treatment in early $(57,58)$ and late (on and after day 7 of onset) $(55,56)$ ARDS confirmed trial-level data demonstrating benefit with corticosteroids, with improved survival and decreased duration of mechanical ventilation (61).

With the exception of hyperglycemia (mostly within the $36 \mathrm{~h}$ following an initial bolus), prolonged glucocorticoid treatment was not associated with increased risk for neuromuscular weakness, gastrointestinal bleeding, or nosocomial infection (61). Hyperglycemia was not associated with increased morbidity. Two trials reported a significant reduction in the risk for developing shock $(56,59)$.

The task force members believed that the quality of the evidence for the effect of corticosteroids on mortality was moderate, given the serious risk of imprecision related to small numbers of events and confidence intervals that approach no effect. Some of the included trials allowed blinded crossover, two trials were unblinded, and four trials had less than 60 patients.

In summary, the task force suggested that methylprednisolone be considered in patients with early (up to day 7 of onset; $\mathrm{PaO}_{2} / \mathrm{FiO}_{2}$ of $<200$ ) in a dose of $1 \mathrm{mg} / \mathrm{kg} /$ day and late (after day 6 of onset) persistent ARDS in a dose of $2 \mathrm{mg} / \mathrm{kg} /$ day followed by slow tapering over 13 days (Supplemental Digital Content 5, http://links.lww.com/CCM/C918). Methylprednisolone is suggested because of its greater penetration into lung tissue and longer residence time (63). Furthermore, methylprednisolone should be weaned slowly (6-14 days) and not stopped rapidly (2-4 days) or abruptly as deterioration may occur from the development of a reconstituted inflammatory response. Finally, glucocorticoid treatment blunts the febrile response; therefore, infection surveillance is recommended to ensure prompt identification and treatment of hospital-acquired infections.

\section{Major Trauma}

Should corticosteroids be administered among hospitalized adult patients with major trauma?

Recommendation: We suggest against the use of corticosteroids in major trauma (conditional recommendation, low quality of evidence).

Rationale: Major trauma is the main cause of non-septic systemic inflammatory response syndrome (SIRS). Tissue necrosis, hemorrhage and ischemia-reperfusion injury are the main factors that trigger the inflammatory cascade. CIRCI may be common in severe trauma patients, and is associated with uncontrolled inflammation, vasopressor dependency and poor clinical outcomes (64).

We found 19 trials $(n=12,269)$ that investigated the effects of corticosteroids on short-term mortality in adults with multiple trauma. There were $1,691 / 6,286(26.9 \%)$ deaths in the corticosteroid group versus 1,401/5,983 (23.4\%) deaths in the placebo group $(\mathrm{RR}=1.00,95 \%$ CI $0.89-1.13)$. Stratified analysis of mortality based on corticosteroid dose (low vs high) found no significant dose effect (test for interaction $p$ $=0.73)$. The RR of dying was 1.03 (95\% CI 0.86-1.22) in the 10 trials that examined low-dose corticosteroid treatment and 0.98 (95\% CI 0.81-1.18) in the nine trials of high-dose corticosteroids. Corticosteroid therapy did not increase the risk of gastroduodenal bleeding ( $n=12$ trials; RR $=1.22,95 \%$ CI $0.90-1.65)$ or superinfection ( $n=7$ trials; $\mathrm{RR}=0.93,95 \% \mathrm{CI}$ 0.80-1.08). Two trials examined the effects of hydrocortisone (65) and hydrocortisone plus fludrocortisone (66) specifically in trauma-associated CIRCI, as defined by a change in baseline cortisol at $60 \mathrm{~min}$ of $<9 \mu \mathrm{g} / \mathrm{dL}$ after cosyntropin $(250$ $\mu \mathrm{g})$ administration. In the first trial $(n=113$ multiple trauma patients with CIRCI), hydrocortisone therapy prevented the development of hospital-acquired pneumonia by day 28 (hazard ratio [HR] 0.47, 95\% CI 0.25-0.86) and increased by 6 days ( $95 \%$ CI 2-11) the number of mechanical ventilation-free days. In the second trial ( $n=267$ head trauma patients with CIRCI), the HR for hospital-acquired pneumonia with corticosteroids versus placebo was 0.80 (95\% CI 0.56-1.14). In this trial, there was no interaction between response to corticosteroid therapy and CIRCI status. See Supplemental Digital Content 4 (http:// links.lww.com/CCM/C917) for evidence profile.

The largest trials which primarily drive the signal for mortality outcome were at low risk of bias, and stratified analysis found no dose effect. Although the type of patients and the formulation, dose, and duration of corticosteroids varied fairly widely across trials, there was no evidence for significant inconsistency in the results. Although it appears that corticosteroids have no effect on mortality in trauma patients, the imprecision of pooled results does not allow exclusion of a potential for benefit or harm from corticosteroid therapy. The task force members judged the overall quality of evidence for this question as low. Given the potential for clinically important side effects with treatment, the task force made a conditional recommendation against corticosteroids for major trauma until further data are available supporting its use.

\section{ACKNOWLEDGMENTS}

We acknowledge the assistance of Jean S. Maragno, Director, Library Services, Sherman Library, St. Joseph's Healthcare Hamilton, Hamilton, Ontario and Lois Cottrell, Librarian, Library Services, Sherman Library, St. Joseph's Healthcare Hamilton, Hamilton, Ontario. We are also grateful to the SCCM staff who helped us during the process of developing these guidelines, particularly Lori A. Harmon, RRT, MBA, Director, Quality and Sylvia Quintanilla, Guidelines Manager. 


\section{REFERENCES}

1. Marik PE, Pastores SM, Annane D, et al: American College of Critical Care Medicine. Recommendations for the diagnosis and management of corticosteroid insufficiency in critically ill adult patients: consensus statements from an international task force by the American College of Critical Care Medicine. Crit Care Med 2008; 36(6):1937-1949

2. Cochrane Collaboration: Cochrane: Review Manager. Edition 5.2. Edited by Centre TNC. Copenhagen, Denmark, Cochrane Collaboration, 2012

3. Shea BJ, Grimshaw JM, Wells GA, et al: Development of AMSTAR: a measurement tool to assess the methodological quality of systematic reviews. BMC Med Res Methodo/ 2007; 7:10

4. Higgins JP, Altman DG, Gøtzsche PC, et al; Cochrane Bias Methods Group; Cochrane Statistical Methods Group: The Cochrane Collaboration's tool for assessing risk of bias in randomised trials. BMJ 2011; 343:d5928

5. Wells GA, Shea B, O'Connell D, et al: The Newcastle-Ottawa Scale (NOS) for assessing the quality of nonrandomised studies in metaanalyses. Available at: www.ohri.ca/programs/clinical_epidemiology/ oxford.asp. Accessed November 21, 2016

6. Whiting P, Rutjes AW, Reitsma JB, et al: The development of QUADAS: a tool for the quality assessment of studies of diagnostic accuracy included in systematic reviews. BMC Med Res Methodo/ 2003; 3:25

7. Kavanagh BP: The GRADE system for rating clinical guidelines. PLoS Med 2009; 6:e1000094

8. Andrews JC, Schünemann HJ, Oxman AD, et al: GRADE guidelines: 15. Going from evidence to recommendation-determinants of a recommendation's direction and strength. J Clin Epidemio/ 2013; 66:726-735

9. Rhodes A, Evans LE, Alhazzani W, et al: Surviving Sepsis Campaign: International Guidelines for Management of Sepsis and Septic Shock: 2016. Intensive Care Med 2017 Mar; 43(3):304-377

10. Bornstein SR, Allolio B, Arlt W, et al: Diagnosis and treatment of primary adrenal insufficiency: An Endocrine Society Clinical Practice Guideline. J Clin Endocrinol Metab 2016; 101(2):364-389

11. Marik PE, Zaloga GP: Adrenal insufficiency during septic shock. Crit Care Med 2003; 31(1): 141-145

12. de Jong MF, Molenaar N, Beishuizen A, et al: Erratum to: Diminished adrenal sensitivity to endogenous and exogenous adrenocorticotropic hormone in critical illness: a prospective cohort study. Crit Care 2015; 19:313

13. Yang $Y$, Liu $L$, Jiang $D$, et al: Critical illness-related corticosteroid insufficiency after multiple traumas: a multicenter, prospective cohort study. J Trauma Acute Care Surg 2014; 76:1390-1396

14. Menon K, Ward RE, Lawson ML, et al; Canadian Critical Care Trials Group: A prospective multicenter study of adrenal function in critically ill children. Am J Respir Crit Care Med 2010; 182:246-251

15. Hamrahian AH, Oseni TS, Arafah BM: Measurements of serum free cortisol in critically ill patients. N Engl J Med 2004; 350:1629-1638

16. Molenaar N, Johan Groeneveld AB, Dijstelbloem HM, et al: Assessing adrenal insufficiency of corticosteroid secretion using free versus total cortisol levels in critical illness. Intensive Care Med 2011; 37:1986-1993

17. Tarjányi Z, Montskó G, Kenyeres $P$, et al: Free and total cortisol levels are useful prognostic markers in critically ill patients: a prospective observational study. Eur J Endocrinol 2014; 171:751-759

18. Jessop DS, Turner-Cobb JM: Measurement and meaning of salivary cortisol: a focus on health and disease in children. Stress 2008; 11:1-14

19. Galbois A, Obadia E, Chalumeau-Lemoine L, et al: Using serum total cortisol assays overstates the adrenal insufficiency prevalence in cirrhotic patients. Eur Rev Med Pharmacol Sci 2016; 20:3730-3731

20. Galbois A, Rudler M, Massard J, et al: Assessment of adrenal function in cirrhotic patients: salivary cortisol should be preferred. $J$ Hepatol 2010; 52:839-845

21. Estrada-Y-Martin RM, Orlander PR: Salivary cortisol can replace free serum cortisol measurements in patients with septic shock. Chest 2011; 140(5):1216-1222

22. Coolens JL, Van Baelen H, Heyns W: Clinical use of unbound plasma cortisol as calculated from total cortisol and corticosteroid-binding globulin. J Steroid Biochem 1987; 26:197-202
23. Ho JT, Al-Musalhi H, Chapman MJ, et al: Septic shock and sepsis: a comparison of total and free plasma cortisol levels. J Clin Endocrinol Metab 2006; 91:105-114

24. Ospina NS, Al Nofal A, Bancos I, et al: ACTH Stimulation Tests for the Diagnosis of Adrenal Insufficiency: Systematic Review and MetaAnalysis. J Clin Endocrinol Metab 2016; 101:427-434

25. Moraes RB, Friedman G, Tonietto T, et al: Comparison of low and high dose cosyntropin stimulation tests in the diagnosis of adrenal insufficiency in septic shock patients. Horm Metab Res 2012; 44:296-301

26. Schneider AJ, Voerman HJ: Abrupt hemodynamic improvement in late septic shock with physiological doses of glucocorticoids. Intensive Care Med 1991; 17:436-437

27. Baldwin WA, Allo M: Occult hypoadrenalism in critically ill patients. Arch Surg 1993; 128:673-676

28. Annane D, Bellissant E, Sebille V, et al: Impaired pressor sensitivity to noradrenaline in septic shock patients with and without impaired adrenal function reserve. Br J Clin Pharmacol 1998; 46:589-597

29. Marik PE, Zaloga GP: Adrenal insufficiency during septic shock. Crit Care Med 2003;31:141-145

30. Annane D, Sébille V, Charpentier C, et al: Effect of treatment with low doses of hydrocortisone and fludrocortisone on mortality in patients with septic shock. JAMA 2002; 288:862-871

31. Arabi YM, Aljumah A, Dabbagh O, et al: Low-dose hydrocortisone in patients with cirrhosis and septic shock: a randomized controlled trial. CMAJ 2010; 182:1971-1977

32. Sprung CL, Annane D, Keh D, et al; CORTICUS Study Group: Hydrocortisone therapy for patients with septic shock. $N$ Engl J Med 2008; 358:111-124

33. Keh D, Trips E, Marx G, et al; SepNet-Critical Care Trials Group: Effect of Hydrocortisone on Development of Shock Among Patients With Severe Sepsis: The HYPRESS Randomized Clinical Trial. JAMA 2016; 316:1775-1785

34. Wira CR, Dodge K, Sather J, Dziura J: Meta-analysis of protocolized goal-directed hemodynamic optimization for the management of severe sepsis and septic shock in the Emergency Department. West J Emerg Med 2014; 15:51-59

35. Annane D, Bellissant E, Bollaert PE, Briegel J, Keh D, Kupfer Y: Corticosteroids for treating sepsis. Cochrane Database Syst Rev 2015; 12:CD002243

36. Angus DC, Linde-Zwirble WT, Lidicker J, Clermont G, Carcillo J, Pinsky MR: Epidemiology of severe sepsis in the United States: analysis of incidence, outcome, and associated costs of care. Crit Care Med 2001; 29(7):1303-1310

37. Martin GS, Mannino DM, Eaton S, Moss M: The epidemiology of sepsis in the United States from 1979 through 2000. N Engl J Med 2003;348(16): 1546-1554

38. Torio CM, Andrews RM: National Inpatient Hospital Costs: The Most Expensive Conditions by Payer, 2011: Statistical Brief \#160. Healthcare Cost and Utilization Project (HCUP) Statistical Briefs [Internet]. Rockville (MD): Agency for Healthcare Research and Quality (US); 2006 Feb. 2013 Aug

39. Fleischmann C, Thomas-Rueddel DO, Hartmann M, et al: Hospital incidence and mortality rates of sepsis. Dtsch Arztebl Int 2016; 113(10):159-166

40. Annane D, Bellisant E, Cavaillon JM: Septic shock. Lancet 2005; 365(9453):63-78

41. Prigent $\mathrm{H}$, Maxime V, Annane D: Clinical review: Corticotherapy in sepsis. Crit Care 2004; 8(2):122-129

42. Annane D, Bellissant E, Bollaert PE, Briegel J, Keh D, Kupfer Y: Corticosteroids for treating sepsis. Cochrane Database Syst Rev 2015; 12:CD002243

43. Gibbison B, López-López JA, Higgins JP, Miller T, Angelini GD, Lightman SL, Annane D: Corticosteroids in septic shock: A systematic review and network meta-analysis. Crit Care 2017; 21(1):78

44. O'Hearn K, McNally D, Choong K, et al; Canadian Critical Care Trials Group: Steroids in fluid and/or vasoactive infusion dependent pediatric shock: study protocol for a randomized controlled trial. Trials 2016; 17:238

45. Menon K, McNally D, O'Hearn K, et al; Canadian Critical Care Trials Group: A Randomized Controlled Trial of Corticosteroids in Pediatric 
Septic Shock: A Pilot Feasibility Study. Pediatr Crit Care Med 2017; 18:505-512

46. Tongyoo S, Permpikul C, Mongkolpun W, et al: Hydrocortisone treatment in early sepsis-associated acute respiratory distress syndrome: results of a randomized controlled trial. Crit Care 2016; 20:329

47. El-Nawawy A, Khater D, Omar H, et al: Evaluation of Early Corticosteroid Therapy in Management of Pediatric Septic Shock in Pediatric Intensive Care Patients: A Randomized Clinical Study. Pediatr Infect Dis J 2017; 36:155-159

48. Annane D, Buisson CB, Cariou A, et al; APROCCHSS Investigators for the TRIGGERSEP Network: Design and conduct of the activated protein $\mathrm{C}$ and corticosteroids for human septic shock (APROCCHSS) trial. Ann Intensive Care 2016; 6:43

49. Ranieri VM, Thompson BT, Barie PS, et al: Drotrecogin alfa (activated) in adults with septic shock. N Engl J Med 2013; 366(22):2055-2064

50. Venkatesh B, Myburgh J, Finfer S, et al: The ADRENAL study protocol: adjunctive corticosteroid treatment in critically ill patients with septic shock. Crit Care Resusc 2013; 15(2):83-88

51. Bellani G, Laffey JG, Pham T, et al; LUNG SAFE Investigators; ESICM Trials Group: Epidemiology, Patterns of Care, and Mortality for Patients With Acute Respiratory Distress Syndrome in Intensive Care Units in 50 Countries. JAMA 2016; 315:788-800

52. Angus DC, Clermont G, Linde-Zwirble WT, et al; NO-06 Investigators: Healthcare costs and long-term outcomes after acute respiratory distress syndrome: A phase III trial of inhaled nitric oxide. Crit Care Med 2006; 34:2883-2890

53. Hill AD, Fowler RA, Burns KE, et al: Long-Term Outcomes and Health Care Utilization after Prolonged Mechanical Ventilation. Ann Am Thorac Soc 2017; 14:355-362

54. Herridge MS, Chu LM, Matte A, et al; RECOVER Program Investigators (Phase 1: towards RECOVER); Canadian Critical Care Trials Group: The RECOVER Program: Disability Risk Groups and 1-Year Outcome after 7 or More Days of Mechanical Ventilation. Am J Respir Crit Care Med 2016; 194:831-844

55. Meduri GU, Headley AS, Golden E, et al: Effect of prolonged methylprednisolone therapy in unresolving acute respiratory distress syndrome: a randomized controlled trial. JAMA 1998; 280:159-165
56. Steinberg KP, Hudson LD, Goodman RB, et al; National Heart, Lung, and Blood Institute Acute Respiratory Distress Syndrome (ARDS) Clinical Trials Network: Efficacy and safety of corticosteroids for persistent acute respiratory distress syndrome. N Engl J Med 2006; 354:1671-1684

57. Meduri GU, Golden E, Freire AX, et al: Methylprednisolone infusion in early severe ARDS: Results of a randomized controlled trial. Chest 2007; 131(4):954-963

58. Rezk NA, Ibrahim AM. Effects of methylprednisolone in early ARDS. Egyptian J Chest Dis \& Tuberc 2013; 6291:167-172

59. Confalonieri M, Urbino R, Potena $A$, et al: Hydrocortisone infusion for severe community-acquired pneumonia: a preliminary randomized study. Am J Respir Crit Care Med 2005; 171:242-248

60. Annane D, Sébille V, Bellissant E; Ger-Inf-05 Study Group: Effect of low doses of corticosteroids in septic shock patients with or without early acute respiratory distress syndrome. Crit Care Med 2006; 34:22-30

61. Meduri GU, Bridges L, Shih MC, et al: Prolonged glucocorticoid treatment is associated with improved ARDS outcomes: analysis of individual patients' data from four randomized trials and trial-level meta-analysis of the updated literature. Intensive Care Med 2016; 42:829-840

62. Meduri GU, Eltorky MA: Understanding ARDS-associated fibroproliferation. Intensive Care Medicine 2015; 41(3): 517-520

63. Greos LS, Vichyanond P, Bloedow DC, et al: Methylprednisolone achieves greater concentrations in the lung than prednisolone. A pharmacokinetic analysis. Am Rev Respir Dis 1991; 144:586-592

64. Hoen S, Asehnoune K, Brailly-Tabard S, et al: Cortisol response to corticotropin stimulation in trauma patients: influence of hemorrhagic shock. Anesthesiology 2002; 97:807-813

65. Roquilly $A$, Mahe PJ, Seguin P, et al: Hydrocortisone therapy for patients with multiple trauma: the randomized controlled HYPOLYTE study. JAMA 2011; 305:1201-1209

66. Asehnoune K, Seguin P, Allary J, et al; Corti-TC Study Group: Hydrocortisone and fludrocortisone for prevention of hospital-acquired pneumonia in patients with severe traumatic brain injury (Corti-TC): a double-blind, multicentre phase 3 , randomised placebo-controlled trial. Lancet Respir Med 2014; 2:706-716 